\title{
DISTAL PANCREATECTOMY IN THE SURGICAL TREATMENT OF PANCREATIC CANCER- A STUDY
}

\author{
Purujit Choudhury 1
}

1Associate Professor, Department of Surgery, Gauhati Medical College, Assam.

\section{BACKGROUND}

Improvements in the outcome of distal pancreatectomy (DP) for cancer is well accepted, but the series were small and heterogeneous. The aim of the present study was to assess perioperative and long-term outcomes of DP for pancreatic adenocarcinoma in the era of multimodal treatment of pancreatic cancer.

\section{MATERIALS AND METHODS}

This was a hospital-based study of all patients undergoing DP for pancreatic adenocarcinoma between 2009 and 2015 . Postoperative and long-term outcomes were assessed retrospectively, and outcome predictors were explored by multivariable analysis.

\section{RESULTS}

A total of 278 patients were enrolled. Multivisceral resections were performed in 58 patients (20 - 9 percent), venous resections in 33 (11 - 9 percent) and arterial resections in 11 (40 percent). Neoadjuvant chemoradiotherapy was used in 20 patients. Postoperative complications occurred in 96 patients (34 - 5 percent) and pancreatic fistulas developed in 76 (27 - 3 percent). The postoperative 90-day mortality rate was $5-0$ percent. In univariable analysis, multivisceral resection was the only factor associated with postoperative morbidity ( $\mathrm{p}=0$ - 048). Age 65 years or less, body mass index of at least $30 \mathrm{~kg} / \mathrm{m}^{2} \mathrm{and}$ absence of preoperative chemoradiotherapy were associated with an increased risk of pancreatic fistula in multivariable analysis. Overall, survival rates at 3 and 5 years were $44-9$ and 29 - 5 percent respectively. In multivariable analysis, only the presence of lymph node metastases was associated with poorer overall survival.

\section{CONCLUSION}

Postoperative morbidity and mortality associated with pancreatic fistula remain considerable after DP, but both short- and longterm survival have improved markedly.

\section{KEYWORDS}

Pancreatectomy, Pancreatic Cancer.

HOW TO CITE THIS ARTICLE: Choudhury P. Distal pancreatectomy in the surgical treatment of pancreatic cancer- a study. J. Evolution Med. Dent. Sci. 2018;7(10):1255-1258, DOI: 10.14260/jemds/2018/286

\section{BACKGROUND}

Distal pancreatectomy (DP) with splenectomy is the standard treatment with curative intent for left-sided pancreatic adenocarcinoma. However, at diagnosis only 12 - 18 percent of patients have a resectable tumour.1,2 Advances in diagnostic tools, operative techniques and perioperative care have improved resectability of such tumours, but reported 5year survival rates after resection remain disappointing, ranging from 10 to 29 percent. ${ }^{3-5}$

The largest available series ${ }^{6-9}$ reporting perioperative and long-term outcome of DP either include tumours other than pancreatic adenocarcinoma such as endocrine tumours or include all pancreatic adenocarcinomas regardless of location. Thus, specific postoperative morbidity and longterm prognosis after DP for pancreatic invasive carcinoma remain poorly studied. The aim of this study was to assess perioperative and long-term outcomes, identifying prognostic factors for long-term survival in a large and hospital-based series of DP for pancreatic adenocarcinoma.

'Financial or Other Competing Interest': None.

Submission 25-01-2018, Peer Review 18-02-2018,

Acceptance 24-02-2018, Published 05-03-2018.

Corresponding Author:

Dr. Purujit Choudhury,

P. O. Gopinath Nagar,

Arya Path, Gauhati-781016, Assam.

E-mail: drpurujit@yahoo.in

DOI: $10.14260 /$ jemds $/ 2018 / 286$

\section{MATERIALS AND METHODS}

A database was built for a national survey on oncological pancreatic surgery. Data was collected by retrospective review of hospital records from January 2009 to December 2015 , and from a prospective survey conducted in 2016. All patients undergoing DP for pancreatic adenocarcinoma in participating centres between 2009 and 2015 were included. No more than three surgeons per centre performed the resections. Demographic data, operative and pathological findings, postoperative and long-term outcomes were collected.

Neoadjuvant chemoradiation was delivered at a total dose of 45 Gy administered in fractions of $1-8$ Gy five times weekly with concurrent chemotherapy by continuous infusion of 5 -fluorouracil (S-FU) $\left(650 \mathrm{mg} / \mathrm{m}^{2}\right.$ on days 1 - 5 and days 21 - 25), supplemented with a cisplatin bolus (80 mg per day per $\mathrm{m}^{2}$ on days 2 and 22) in two centres. Neoadjuvant chemotherapy was based on either 5-FUleucovorin or 5-FU-oxaliplatin (FOLFOX) regimens and lasted from 3 to 6 months. Intraoperative data included concomitant splenectomy, extended visceral and/or vascular resections, texture of pancreatic parenchyma (graded by the surgeon as soft, normal or hard), site of pancreatic transaction (neck versus body), technique of suture of the pancreatic remnant (stapler or suture), perioperative transfusions and prophylactic use of somatostatin analogues. Multivisceral resections were defined as simultaneous resections of organs 
other than pancreas and spleen (including colon, kidney, liver, adrenal gland and stomach).

Postoperative mortality in terms of all deaths occurring during the first 90 days after surgery were looking into account. Major and minor postoperative complications were classified according to the Dindo-Clavien classification.10 Pancreatic fistulas (PF) were defined and graded according to International consensus as any amylase-rich fluid (Amylase concentration greater than 3 times the serum concentration) after postoperative day 3 , either collected by postoperative puncture of an abdominal collection or in the effluent of surgical drains. ${ }^{11}$ In addition, intra-abdominal collections or abscesses in the absence of any other proven anastomotic leak were considered as secondary to PF. Postoperative bleeding was defined according to International consensus. Oncological quality of resection was established by both the surgeon (for R2 resections) and the pathologist (for R0 or R1 resections). Because of the retrospective design of the study, patients were vaccinated against pneumococcus, either before or after surgery (minimum delay of 4 weeks after surgery) and there was no strict standardisation of surgical approach or technique (Exploratory laparoscopy performed at the same session before definitive open surgery, use of radical antegrade modular resection, technique of pancreatic stump closure, drainage) or of pathological examination. Thus, inking of the retroperitoneal margin and radial margin assessment were not done systematically. An R1 margin was defined as tumour-free margin of less than $1 \mathrm{~mm}$.

Considering the lack of available consensus in the literature about the minimum number of lymph nodes to be assessed in DP for pancreatic adenocarcinoma, all patients without evidence of lymph node metastasis were classified as NO, whatever the number of examined nodes. When used adjuvant therapy lasted 6 months and was based on gemcitabine or 5-FU and leucovorin according to available evidence.

\section{Statistical Analysis}

Results are presented as median (range) unless indicated otherwise. Univariable associations between clinicopathological variables, surgical data and outcome parameters were examined using the $\mathrm{X}$-square test and/or logistic regression analysis as appropriate. Factors independently associated with complications, occurrence of PF and mortality were identified by multiple logistic regression analysis that included all variables with $\mathrm{p}<0.100$ in univariable analysis. Survival curves were constructed using the Kaplan-Meier method, and compared by means of the logrank test. Overall, survival was calculated from the date of pancreatic resection. All deaths from any cause were included in this analysis. Two-sided statistical tests were used and a difference was considered statistically significant at $\mathrm{p}<0.050$. Patients with missing data were excluded from analyses for each of the studied criteria and number of patients included in the analyses are shown. Statistical analysis was performed with SPSS $\AA$ for Windows $\AA$ version 20.0 (IBM, Armonk, New York, USA).

\section{RESULTS}

A total of 301 patients who had DP were enrolled in the study. Twenty-three patients were excluded, because DP was undertaken for tumours other than pancreatic adenocarcinoma. Data for the remaining 28 patients (median age $64(22-90)$ years, who were treated by DP for invasive pancreatic ductal adenocarcinoma were analysed (Table 1).

\section{Intraoperative and Pathological Findings}

Exploratory laparoscopy was performed in 45 patients (16 - 2 percent) followed immediately by an attempt at laparoscopic resection in 15 patients with conversion.

\begin{tabular}{|c|c|c|}
\hline & No. of Patients & No. of Patients \\
\hline Age $>65$ years & 134 of $276(48-6)$ & $\mathrm{n}=254$ \\
\hline \begin{tabular}{|c|} 
Body mass index $>30$ \\
$\mathrm{~kg} / \mathrm{m}^{2}$
\end{tabular} & 21 of $225(9-3)$ & $35(13-7)$ \\
\hline Men & 125 of $278(450)$ & $219(86-3)$ \\
\hline $\begin{array}{c}\text { ASA fitness grade III or } \\
\text { IV }\end{array}$ & 42 of $238(17-6)$ & $n=227$ \\
\hline Diabetes & 47 of $255(18-4)$ & $39(17-2)$ \\
\hline Neoadjuvant treatment & $n=278$ & $188(82-8)$ \\
\hline Chemotherapy & $19(6-8)$ & $\mathrm{n}=261$ \\
\hline Chemoradiation & $20(7-2)$ & $2(0-8)$ \\
\hline $\begin{array}{c}\text { Centre volume } \\
(<10 \text { resections/year })\end{array}$ & 103 of $278(37-1)$ & $20(7-7)$ \\
\hline Type of resection & $\mathrm{n}=278$ & $38(14-6)$ \\
\hline DP + splenectomy & $254(914)$ & $166(63-6)$ \\
\hline SPDP & $24(8-6)$ & $35(13-4)$ \\
\hline Pancreatic consistency & $n=224$ & $\mathrm{n}=260$ \\
\hline Soft & $29(12-9)$ & $109(41-9)$ \\
\hline Normal & $136(60-7)$ & $151(58-1)$ \\
\hline Fibrous/ Hard & $59(26-3)$ & $\mathrm{n}=259$ \\
\hline Arterial resection* & 11 of $278(4-0)$ & $37(14-3)$ \\
\hline Venous resection* & 33 of $260(12-7)$ & $153(59-1)$ \\
\hline Multivisceral resection & 58 of $263(22-1)$ & $25(9-7)$ \\
\hline $\begin{array}{l}\text { Intraoperative RBC } \\
\text { transfusion }>2 \text { units }\end{array}$ & 12 of $241(5-0)$ & $44(17-0)$ \\
\hline $\begin{array}{l}\text { Pancreatic remnant } \\
\text { closure }\end{array}$ & $n=247$ & $\mathrm{n}=247$ \\
\hline Stapler & $54(21-9)$ & $128(51-8)$ \\
\hline Suture & $193(78-1)$ & $119(48-2)$ \\
\hline \begin{tabular}{|c|} 
Drainage of pancreatic \\
stump
\end{tabular} & 260 of 273 (95.2) & $\mathrm{n}=244$ \\
\hline
\end{tabular}

ASA- American Society of Anesthesiologists; DP- Distal pancreatectomy; SPDR- Spleen-preserving distal pancreatectomy; RBC- Red blood cell.

Values in parentheses are percentages. Information on studied criteria was not available for all patients. Resection of coeliac trunks (8 patients), hepatic arteries (2) and superior mesenteric artery (1). Resection of portal or superior mesenteric veins to laparotomy in six. The spleen was preserved in 24 patients $(8-6 \%)$. In half of these spleenpreserving DPs, the tumour was located in the central part of the pancreas. Multivisceral resections were carried out in 58 patients (20 - 9 percent) to achieve macroscopically complete resection. In 26 of these 58 patients, two or more organs (other than pancreas) were involved. Vascular resections were performed in 39 patients. There were 33 venous resections (11 - 9\%) of portal and/or superior mesenteric vein and 11 arterial resections (4\%); 8 coeliac trunk resections during Appleby procedures preserving the stomach and performed without preoperative hepatic artery embolisation, 2 hepatic arteries, 1 superior mesenteric artery (Table 1). Five patients had concomitant arterial and venous resections. 
Two of the 20 patients who underwent neoadjuvant chemoradiotherapy had a complete pathological response (Table 2). The median number of examined lymph nodes was 17 (0 - 46\%); $27.1 \%$ of pathological examinations reported fewer than ten examined nodes per patient. The median number of lymph nodes harvested was 8 (range 0 - 22\%) when the spleen was preserved significantly.

\begin{tabular}{|c|c|}
\hline & No. of Patients \\
\hline Tumour Size $(\mathrm{cm})$ & $\mathrm{n}=254$ \\
\hline$<3-0$ & $35(13-7)$ \\
\hline$>3-0$ & $219(86-3)$ \\
\hline Tumour Differentiation & $\mathrm{n}=227$ \\
\hline Poor & $39(17-2)$ \\
\hline Moderate/Well & $188(82-8)$ \\
\hline Tumour Category & $\mathrm{n}=261$ \\
\hline T0 & $2(0-8)$ \\
\hline T1 & $20(7-7)$ \\
\hline T2 & $38(14-6)$ \\
\hline T3 & $166(63-6)$ \\
\hline T4 & $35(13-4)$ \\
\hline Nodal Status & $\mathrm{n}=260$ \\
\hline N0 & $109(41-9)$ \\
\hline N1 & $151(58-1)$ \\
\hline TNM Stage & $\mathrm{n}=259$ \\
\hline 1 & $37(14-3)$ \\
\hline II & $153(59-1)$ \\
\hline III & $25(9-7)$ \\
\hline IV & $44(17-0)$ \\
\hline Vascular Emboli & $\mathrm{n}=247$ \\
\hline Yes & $128(51-8)$ \\
\hline No & $119(48-2)$ \\
\hline Microscopic & $\mathrm{n}=244$ \\
\hline Perineural Invasion 2 Pathological Data \\
\hline
\end{tabular}

Values in parentheses are percentages. Information on histological characteristics was not available for all patients. "International Union against Cancer/ American Joint Committee on Cancer, 7th edition 0009).

Lower than the median of 15 (range 4 - 46) in operations with associated splenectomy $(\mathrm{p}=0-002)$, but this had no significant effect on overall survival.

\section{Postoperative Morbidity and Mortality}

Ninety-six patients (34 - 5\%) developed postoperative complications. PF prophylaxis with somatostatin or analogues was used in 96 (38 - 1 percent) of 252 patients for whom such data were available. PF occurred in 76 patients (27 - 3 percent). Its incidence was not reduced by the use of somatostatin (Analogue) prophylaxis $(\mathrm{p}=0$ - 485). In 45 patients (16 - 2\%), complications (whether medical or surgical) were classified as major (Dindo-Clavien grade III or more). Postoperative complications required re-laparotomy in 21 patients ( 7 - 6 percent). Postoperative bleeding and $\mathrm{PF} /$ pancreatic abscess formation were the most frequent indications for reoperation. Postoperative intra-abdominal bleeding requiring specific intervention was observed in 12 patients (4 - 3\%); surgical and radiological embolisations were carried out in five and seven patients respectively. Two patients died in the first postoperative month $(0-7$ percent $)$ and the 90 -day mortality rate was $5-0 \%$ (14 deaths). One of the 11 patients who underwent arterial resection died after surgery.
Univariable logistic regression analysis failed to identify any preoperative or intraoperative factors associated with 90 -days mortality. The need for a multivisceral resection was the only factor associated with postoperative morbidity in univariable analysis (Odds ratio 1-82, 95 percent, CI 1 - 00 to 3 - 32; $p=0$ - 048). Age 65 years or less, body mass index over $30 \mathrm{~kg} / \mathrm{m}^{2}$ and absence of preoperative radiotherapy were associated with an increased risk of pancreatic fistula in univariable and multivariable analyses.

\section{Long-Term Outcome}

All 261 patients with follow-up of 90 days or more were included in univariable and multivariable analyses of overall survival. Median overall survival was 35 (95 percent, CI 29-5 to 40-5) months. During follow-up, 86 patients died after a median survival of 12 (range 3 - 57) months, whereas 175 patients were alive with a median follow-up of 13 (range 367) months. Overall survival rates at 1,2, 3 and 5 years were $80-2,59-6,44-9$ and $29-5 \%$ respectively.

One hundred and eighty-six patients (71 - 3 percent) were treated with adjuvant chemotherapy. Among these, the chemotherapy regimen was specified in 175 patients and was mostly gemcitabine-based (147, 84 - 0 percent).

In univariable analyses, age over 65 years, male sex, intraoperative red blood cell blood transfusion of at least 2 units, multivisceral resection, tumour size of $4 \mathrm{~cm}$ or more, $\mathrm{T}$ category (International Union against Cancer/American Joint Committee on Cancer, 7th edition, 2009,) lymph node metastases and poor histological differentiation were significantly associated with poorer overall survival. In multivariable analysis only the presence of lymph node metastases remained independently associated with poorer overall survival. An R1 resection did not significantly alter overall survival $(\mathrm{p}=0$ - 138).

\section{DISCUSSION}

The present multicentre study collected data on DP for pancreatic cancer outcomes were compared with those of a similar western survey conducted about 20 years ago. Postoperative morbidity in the present series was similar to that reported previously, whereas reoperation rates and mortality rates improved dramatically (now 5\% - 0\% at 90 days versus $9 \%$ at 30 days in the previous survey.) There are now long-term survivors with an acceptable 5-year survival rate of $29-5 \%$. The $\mathrm{PF}$ rate $(27-3 \%)$ and risk factors for fistula were comparable to those in previous series. Obesity was significantly associated with PF formation in another study. The lower rate of PF after preoperative radiotherapy in the present series confirms published findings. The higher risk of PF in patients less than 65 years old might result from their greater pancreatic stump volume. Neither diabetes, soft consistency of the pancreas, nor the use of staplers for pancreatic closure significantly affected PF rates, in keeping with a recent randomised trial that showed no benefit from use of staplers compared with hand sewn closure after DP. Finally, in contrast to a recent meta-analysis, the prophylactic use of somatostatin analogues in $38.1 \%$ of patients was not associated with a lower PF rate.

Improved medical and radiological management of postoperative complications is likely to explain the reduced postoperative mortality, which did not result from inclusion 
of expert centres only. Indeed, 10 of 28 participating centres performed fewer than ten pancreatic resections per year (DP and duodenopancreatectomies included). Median overall survival after DP reached 35 months here, compared with 9 months in the previous survey, and 1-, 2-, 3- and 5-year survival rates were $80.2,59.6,44.9$ and $29.5 \%$ respectively versus $38,24,12$ and $0 \%$. These better long-term results might be related to improved patient selection by imaging resulting in tumours frequently being smaller than $4 \mathrm{~cm}$ (36.6\% versus $29 \%$ ), or by progressive adherence by most European pancreatic surgeons to the oncological principles of the radical anterograde modular resection, with more frequent vascular and multivisceral resections and more microscopically complete (R0) resections. Alternatively, better management of surgical complications and wider use of more potent adjuvant therapies or neoadjuvant treatments might explain the better results. No preoperative criteria emerged from this series to contraindicate resection of a resectable tumour. Involvement of en bloc resected adjacent organs was not related to overall survival in multivariable analysis and did not preclude long-term survival. Likewise, resected macroscopic venous involvement of the mesentericoportal axis did not affect survival. Arterial resections (Coeliac trunk or superior mesenteric artery) were performed too rarely in this series to draw reliable conclusions. Nodal involvement was the only independent predictor of poor overall survival as in other series. However, lymphatic involvement is rarely diagnosed before surgery and does not preclude some instances of long-term survival.

Owing to the multicentre and retrospective design of this study, the accuracy of preoperative radiological assessment was not evaluable, and underestimation of postoperative morbidity was possible. Thus, nearly 30 percent rate of PF might actually reflect the incidence of grade $\mathrm{B}, \mathrm{C}$ or D PF. The rates of $\mathrm{N} 1$ and $\mathrm{R} 1$ resections were also probably underestimated, because of the lack of a systematic protocol for nodal assessment and inking of pancreatic margins. Because of the short period of inclusion, only 3-year actuarial estimations of survival rates are likely to be reliable. The value of preoperative diagnostic laparoscopy which was rarely performed and of splenectomy which was carried out almost systematically could not be studied in this series. Finally, the place of laparoscopic DP, more frequently performed now-a-days could not be assessed as this series included mostly open surgery.

\section{CONCLUSION}

Distal pancreatectomy with splenectomy is the commonest procedure in left-sided pancreatic adenocarcinoma, though in a very small percentage of cases is resectable at the time of diagnosis. Lesion with lymph nodes positivity shows poorer prognosis. Multimodal treatment gives better prognosis than surgery alone. Postoperative morbidity and mortality associated with pancreatic fistula remain considerable after DP, but both short and long-term survival have improved markedly.

\section{REFERENCES}

[1] Wagner M, Redaelli C, Lietz M, et al. Curative resection is the single most important factor determining outcome in patients with pancreatic adenocarcinoma. Br J Surg 2004;91(5):586-94.

[2] D'Souza MA, Shrikhande SV. Pancreatic resectional surgery: an evidence-based perspective. J Cancer Res Ther 2008;4(2):77-83.

[3] Schmidt CM, Powell ES, Yiannoutsos CT, et al. Pancreaticoduodenectomy: a 20-year experience in 516 patients. Arch Surg 2004;139(7):718-25.

[4] Bassi C, Falconi M, Salvia R, et al. Management of complications after pancreaticoduodenectomy in a high volume centre: results on 150 consecutive patients. Dig Surg 2001;18(6):453-7.

[5] Balcom JH, Rattner DW, Warshaw AL, et al. Ten-year experience with 733 pancreatic resections: changing indications, older patients, and decreasing length of hospitalization. Arch Surg 2001;136(4):391-8.

[6] Nathan $\mathrm{H}$, Wolfgang CL, Edil BH, et al. Peri-operative mortality and long-term survival after total pancreatectomy for pancreatic adenocarcinoma: a population-based perspective. J Surg Oncol 2009;99(2):87-92.

[7] Christein JD, Kendrick ML, Iqbal CW, et al. Distal pancreatectomy for resectable adenocarcinoma of the body and tail of the pancreas. J Gastrointest Surg 2005;9(7):922-7.

[8] Reeh M, Nentwich MF, Bogoevski D, et al. High surgical morbidity following distal pancreatectomy: still an unsolved problem. World J Surg 2011;35(5):1110-7.

[9] Kleeff J, Diener MK, Z'Graggen $K$, et al. Distal pancreatectomy: risk factors for surgical failure in 302 consecutive cases. Ann Surg 2007;245(4):573-82.

[10] Dindo D, Demartines N, Clavien PA. Classification of surgical complications: a new proposal with evaluation in a cohort of 6336 patients and results of a survey. Ann Surg 2004;240(2):205-13.

[11] Bassi C, Dervenis C, Butturini G, et al. Postoperative pancreatic fistula: an international study group (ISGPF) definition. Surgery 2005;138(1):8-13. 